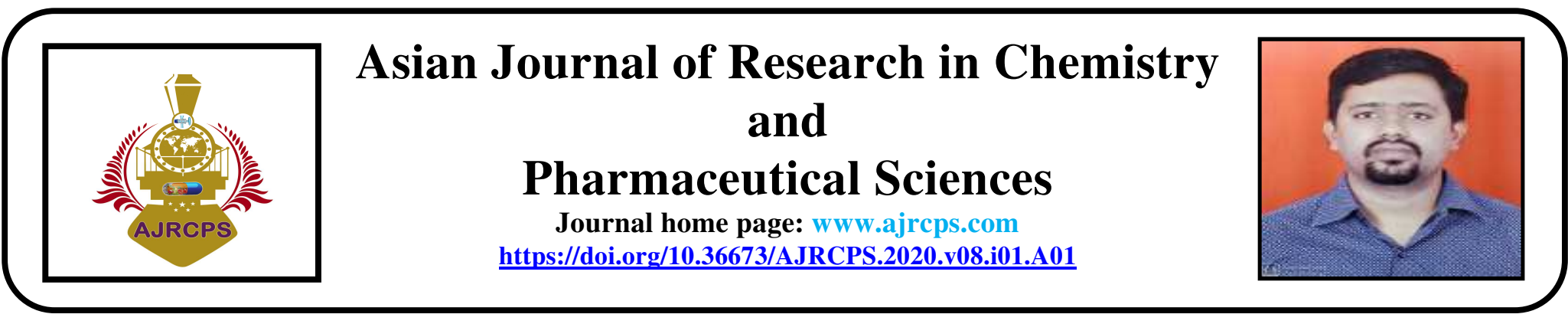

\title{
TABLET IN TABLET: A REVIEW
}

\author{
Mahesh Dattatray Dokhe $^{* 1}$, R. Kunkulol ${ }^{2}$, D. Nandal ${ }^{2}$ \\ 1*Department of Quality Assurance Techniques, Dr. Vitthalrao Vikhe Patil Foundation's College of Pharmacy, \\ Viladghat, Ahmednagar - 414111, Maharashtra, India. \\ ${ }^{2}$ Department of Pharmacology, Pravara Rural College of Pharmacy, Loni BK Tal. Rahata, Dist. Ahmednagar - \\ 413736, Maharashtra, India.
}

\begin{abstract}
In the past some years the number of products based on new drug delivery system has significantly increased, and this extension is expected to continue in the future. Tablet dosage form is convenient and relevant as compared to other dosage forms. Innovation in tablet dosage form provide product of higher selectivity for the drug for medical treatment. There are so many existing drug delivery technologies in recent years developed. This is an attempt to being made to compile some of the most successfully marketed drug delivery technology in this article. The present review focus on innovation in tablet system i.e. Tablet in tablet system. Evolution of an existing drug molecule from a conventional form in to said technology can improve performance in terms of safety, efficacy and patient compliance.
\end{abstract}

\section{KEYWORDS}

Tablet in tablet technology, Sustained release tablet and Immediate release tablet.

\section{Author for Correspondence:}

Mahesh Dattatray Dokhe,

Department of Quality Assurance Techniques,

Dr. Vitthalrao Vikhe Patil Foundation's College of Pharmacy, Viladghat,

Ahmednagar, Maharashtra, India.

Email: mahesh.dokhe1989@gmail.com

\section{INTRODUCTON ${ }^{1,2}$}

Oral route is the most commonly preferred route of drug administration. The popularity of the oral route is due to patient acceptance, ease of administration, accurate dosing and cost effectiveness. Even for sustained release the oral route of administration investigated the most.

The excipient includes binders, diluents, glidants, granulating agents and lubricants for an efficient tablet. Polymer coating is often applied to make tablet easier to swallow and to control the release of the active ingredients.

January - March 
Mahesh Dattatray Dokhe. et al. /Asian Journal of Research in Chemistry and Pharmaceutical Sciences. 8(1), 2020, 1-6.

The main reasons behind formulations of different types of tablets are to create a delivery of drug that is simple and inexpensive to manufacture.

Tablets are vary in different shapes, sizes, and weight, depend upon amount of active pharmaceutical ingredient and mode of administration. Near about $70 \%$ of the total medicines are dispensed in the form of tablet dosage form.

The Advantages of the Tablet Dosage Form ${ }^{3,4}$

Accuracy of dose is maintained as tablet is unit dosage form.

Tablet is not a sterile dosage form, special environmental conditions are not required in tablet department.

Large scale manufacturing is feasible as compare to other dosage forms.

Organoleptic properties are improved by coating of tablet.

The longer expiry period and minimum microbial spillage often due to lower moisture content.

Different types of tablets are available such as buccal, floating, effervescent, dispersible, soluble, mouth dissolving, chewable, etc.

Ease of packaging and easy handling.

Self-administration is possible

Economic than other dosage forms.

Lighter and compact than other oral dosage forms.

Sustained release product is possible by using polymers.

\section{Disadvantages of tablet dosage form ${ }^{5,6}$}

- Difficult to swallow in case of children, geriatrics and unconscious patients.

- Slow onset of action as compare to parenterals, capsules and liquid orals.

- Drug with poor wettability, slow dissolution difficult to formulate into tablet.

- Some drugs resists compression into dense campacts.

- Drugs with bitter taste, objectionable odor or drugs sensitive to oxygen requires encapsulation or coating.

Available online: www.uptodateresearchpublication.com
List of Some Tableting Techniques Currently Used By Pharma Companies ${ }^{7,8}$

1. OSDrC tablet concept (one step dry compression coating)

2. Accu-break technology for controlled release tablets

3. DiffCORE technology for modified release tablets

4. The GEOMATRIX technology

5. Tablet in Tablet technology

Tablet in Tablet Technology $\mathbf{y}^{6,9}$

Generally two type of techniques are used to prepare tablet in tablet formulation

- Inlay Tablets Techniques

- Tablet in Tablet Techniques

\section{Inlay Tablets}

A type of layered tablet in which instead the core tablet being completely surrounded by coating, top surface is completely exposed. (Figure No.1).

In the manufacturing process only the bottom of the die cavity is filled with coating material (cup) and core placed upon it, when compression force is applied the coating material is displaced to compress whole tablet

Tablet in Tablet Techniques ${ }^{10,11}$

In this type of tableting technique the internal core tablet is completely surrounded by external surrounding coat. This tablet has two parts, internal one as core and outer one coating. It looks like a single tablet from outer view but it contains another tablet inside that tablet. This technology gained more interest from some year back for modified released products. It involves compression of granular material around a core tablet i.e. inner core tablet. The tablet in tablet formulation protects the hygroscopic, oxidative, photo sensitive and acidlabile drugs. Compression coating is relatively easy and economic. Compression of tablets is easy on laboratory scale and for large scale manufacturing special equipment is needed. It is more acceptable to the industry, because this system uses conventional manufacturing process (Figure No.2).

Advantages of Tablet in Tablet Technique ${ }^{12,13}$

- The tablet in tablet technique provides initial drug release to provide rapid onset of action

January - March

2 
Mahesh Dattatray Dokhe. et al. /Asian Journal of Research in Chemistry and Pharmaceutical Sciences. 8(1), 2020, 1-6.

followed by a period of sustained release in quick or slow system.

- Reduce dosage frequency.

- The system allow loading of higher amount of drug.

- Administration of two different drugs in one dosage form is possible.

- Administration of two incompatible drug can possible.

- Improved patient compliance.

- Separation of layers not occurs as in case of bilayer tablet dosage form.

- Can avoid contact between two incompatible drugs.

- The combination of immediate release and delayed release dosage form can formulate in single tablet.

Disadvantages of Tablet in Tablet Technique ${ }^{12}$

- Manufacturing is complicated.

- Cost of product is high.

Manufacturing Process of Tablet in Tablet Technology ${ }^{13,14,15}$

This type of layered compression coating tablet has two parts, inner core part and outer surrounding coat. The core is a small tablet which is compressed at first. For preparing a final tablet the bigger die is used. The die cavity is half filled by surrounding material and the core tablet is placed upon it, the remaining coating material is filled over the core tablet in die and compression force is applied to form a final tablet. (Figure No.3)

The outer layer of tablet provides an initial dose while the inner tablet provides a sustained release of drug or provides a release of drug later on. But when the core quickly release the drug there is risk of over dose toxicity. To avoid the burst effect the core tablet is coated with enteric polymer and the outer layer of tablet provides initial dose of drug. The tablet in tablet system can be prepared by direct compression of granules, with flat punches and dies.

\section{EVALUATION OF TABLET IN TABLET ${ }^{16-19}$}

The following quality control tests should carried out on compression coated tablet.
General Appearance

- Size and Shape

- Unique identification marking

- Organoleptic properties

Mechanical Strength of Tablet

1. Hardness

2. Friability

Content Uniformity

1. Weight variation test

2. Disintegration test

3. Dissolution test

\section{General Appearance}

The tablets identity and elegance is essential for acceptance from consumer. The measurement of size, shape, taste, color, odor etc. are involves in control of general appearance.

\section{Size and shape}

The size and shapes of tablet can be dimensionally described and controlled. The thickness of tablet can be variables. The thickness of tablet can be measured by vernier caliper or by other device. Tablet thickness should be controlled within $+/-5 \%$ variation of standard value.

\section{Unique identification marking}

This form of marking includes the debossing, imbossing or printing on the tablet dosage form. The unique identification marking includes company name or symbol, product name or sometimes products code etc.

\section{Organoleptic properties}

The organoleptic properties involves observation of color, odor, taste, appearance etc. the color distribution should be uniform without mottling effect. The color, odor, tastes comparison carried out by comparing with the standard sample.

\section{Mechanical Strength of Tablet}

The mechanical strength of tablet is determine mainly of by two tests as follow

\section{Hardness}

The tablet requires a certain amount of hardness to with stand mechanical shakes during handling, manufacturing, packing and shipping. Tablet crushing strength generally known as hardness of tablet.

January - March 


\section{Friability}

The friability of a tablet can determine be determine by Roche friabilator in laboratory. The Roche friabilator consist of a plastic chamber which revolves at $25 \mathrm{rpm}$ and dropping the tablets from a height of 6 inches in friabilator chamber, which is operate for 100 revolutions. The tablets are reweighted. The acceptable weight loss from tablet to pass the test is less than 0.1 to $0.5 \%$.

\section{Content Uniformity}

The selection of 30 tablets randomly carried out. Out of the 30 tablets 10 tablets individually assayed for content uniformity. If 9 of the 10 tablets must contain not less than $85 \%$ and not more than $115 \%$ and the $10^{\text {th }}$ tablet may not contain less than $75 \%$ or not more than $125 \%$ of the labeled drug content the result is acceptable. If the condition is not met then remaining 20 tablets assayed individually.

\section{Weight variation test (U.S.P.)}

In this test 20 tablets are weighed individually, calculation of average weight and comparison of individual tablets weight to the average one tablet weight is carried out. When not more than 2 tablets are outside the percentage limit the result is acceptable.

\section{Disintegration Test}

The disintegration test is carried out by disintegration test apparatus a U.S.P. device. The device includes 6 glass tubes which are of 3" long with \#10 at bottom and open at the top. Carry a disintegration test one tablet is placed in each tube and basket is positioned in beaker of water, simulated gastric fluid or simulated intestinal fluid at $37+/-2^{\circ} \mathrm{C}$.

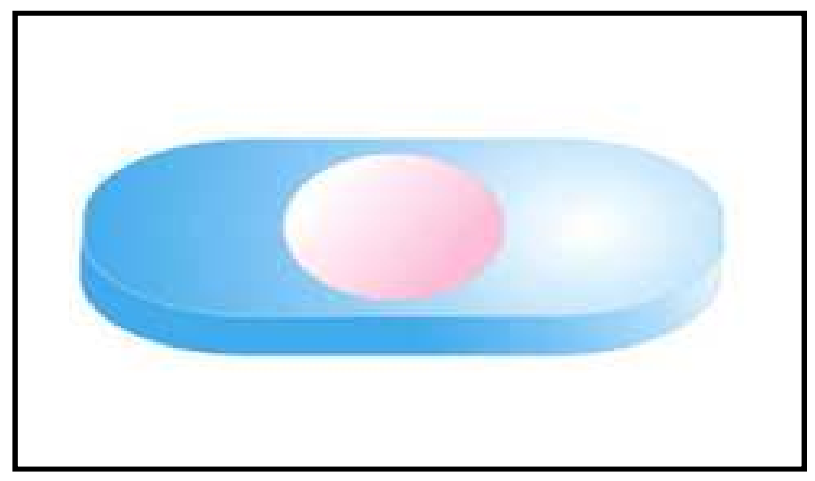

Figure No.1: Inlay Tablet

Available online: www.uptodateresearchpublication.com
Movement of the basket containing the tablets up and down through a distance of 5-6 $\mathrm{cm}$ at a frequency of 28-32 cycles per minute. By placing a perforated plastic discs on each tablets to prevent a floating of the tablet during a test. According to tests tablet must disintegrate and all particles of disintegrated tablet must pass through a \#10 mesh which is at the bottom of the tubes, and if any residue of tablet is remain it should be a soft mass. For uncoated tablet 5-30 minute and for coated tablet 1-2 hours disintegration time requires.

When the tablet in tablet technique contains outer layer as immediate release the disintegration test is carried out for the outer layer of the tablet.

\section{DISSOLUTION TEST}

\section{Apparatus-1 (Basket type)}

A small wire mesh basket attached to the bottom of the shaft connected to a variable speed motor. The motor adjusted to turn at specified speed. A single tablet is placed in basket immersed in a dissolution medium as specified in monogragh contained in $1000 \mathrm{ml}$ flask. The flask is maintained at $37+/-0.5^{\circ} \mathrm{C}$ by constant temperature bath. The fluid are withdrawn at intervals determine the amount of drug released in solutions.

\section{Apparatus-2 (Paddle type)}

This type of apparatus is same as apparatus-1, except the basket is replaced by a paddle. The tablet is allowed to sink at the bottom of the flask before starting a test. The dissolution test medium and volume, type of apparatus used, rpm of the shaft, and time limit of the test is specified in U.S.P. for dissolution test.
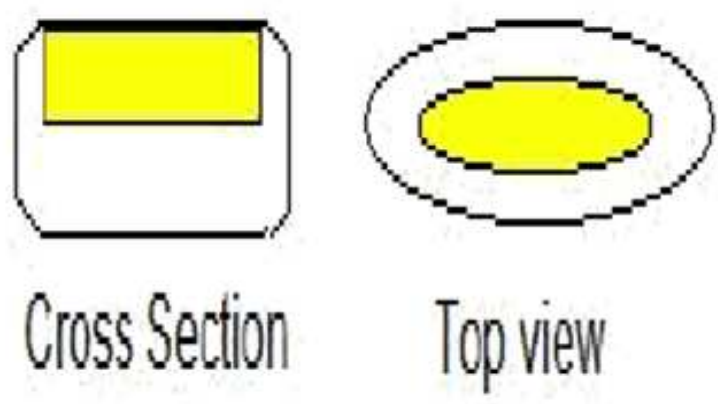

January - March 
Mahesh Dattatray Dokhe. et al. /Asian Journal of Research in Chemistry and Pharmaceutical Sciences. 8(1), 2020, 1-6.

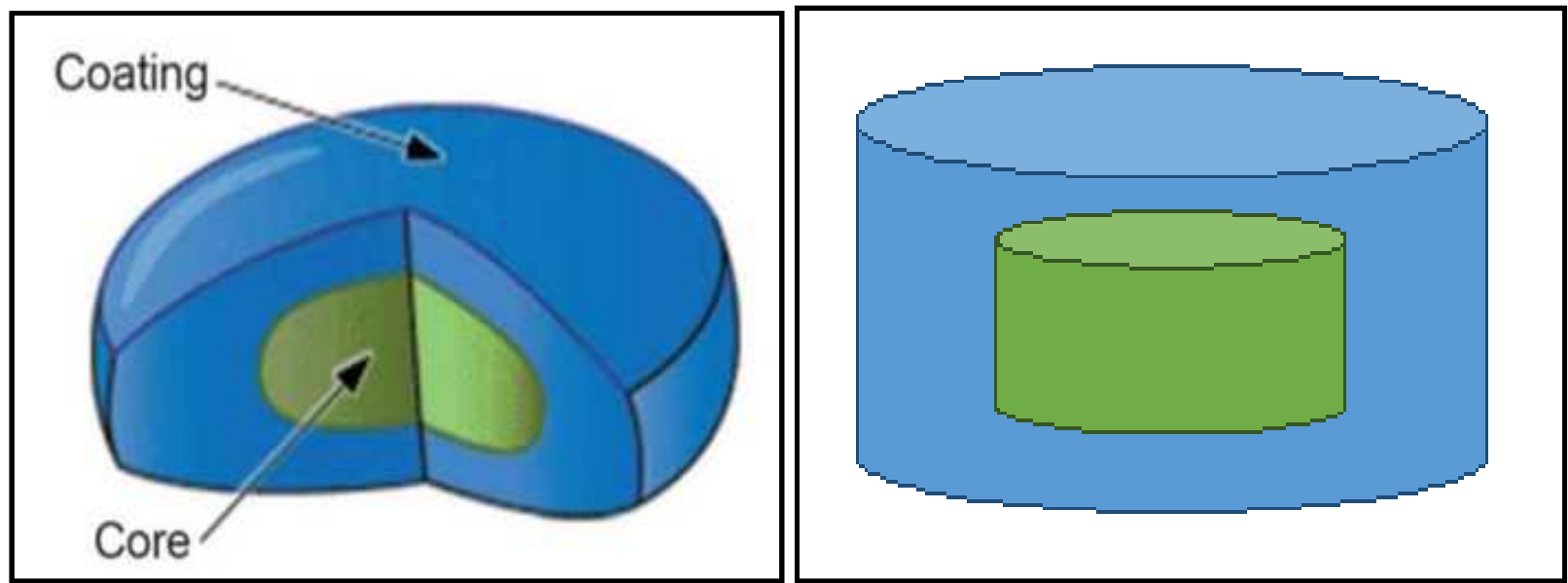

Figure No.2: Tablet in Tablet

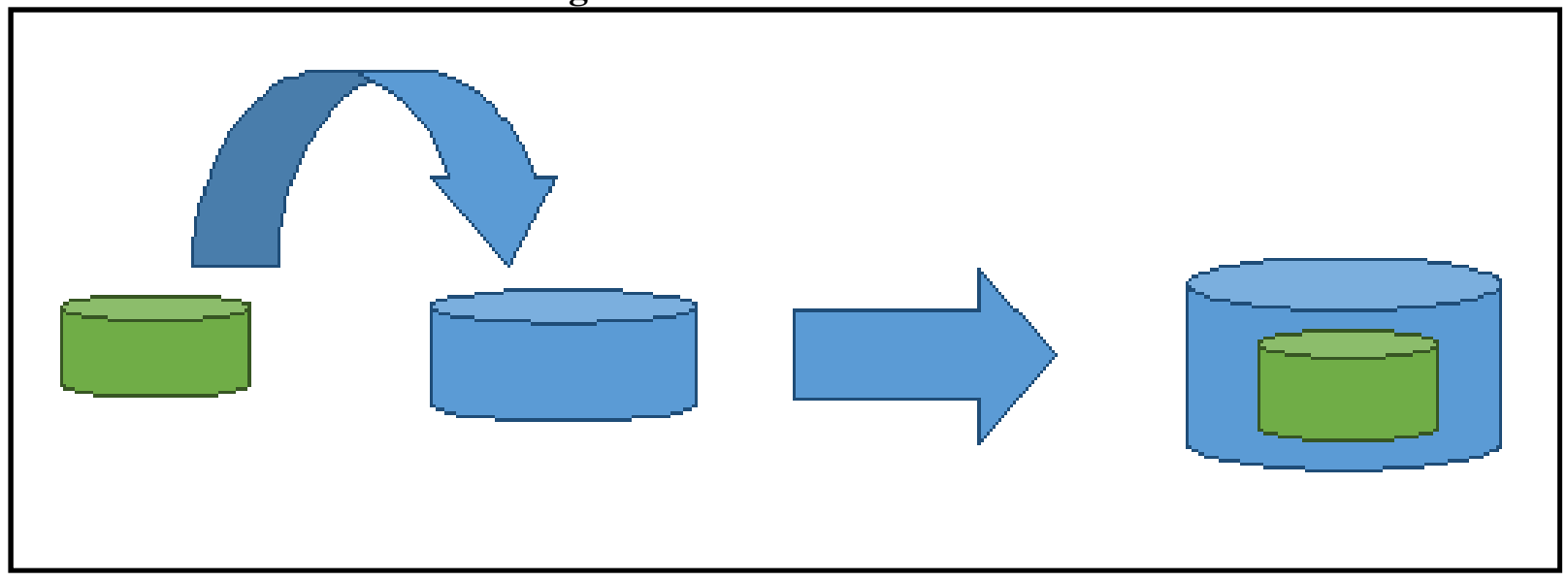

Figure No.3: Manufacturing process of Tablet in Tablet

\section{CONCLUSION}

The tablet in tablet dosage form comprising of an active ingredient of core tablet as sustained release and outer layer as immediate release with the ability to release soluble and insoluble drugs at zero-order rate of release in a dissolution media. The drugs with frequency of high water solubility can be reduced with same efficacy. Problems like frequent dosing, drug interactions, and burst effect can be reduced. Any combination of drug is used with no interactions.

\section{ACKNOWLEDGMENT}

The authors are thankful to their families and friends for their support and encouragements and D.V.V.P. F's College of Pharmacy, Viladghat, Ahmednagar, MH, India for providing facilities to carry out this work.

\section{CONFLICT OF INTEREST}

We declare that we have no conflict of interest.

\section{BIBLIOGRAPHY}

1. Patel B P, Patel R P. A Review on Advances in Tableting Technique, European journal of biomedical and pharmaceutical sciences, 2, 2015.

2. Rajalakshmi R, Sireesha A, Mohana Lakshmi S. Inlay Tablets- A Novel Approach, International journal of advanced pharmaceutics, 1(1), 2011, 1-10.

3. Preetha P, Shrinivasa Rao A, Pushpalatha P. Biphasic Drug Delivery In Controlled Release Formulations- A Review, International Journal of Pharmacy and Technology, 6(4), 2015, 3046-3060. 
Mahesh Dattatray Dokhe. et al. /Asian Journal of Research in Chemistry and Pharmaceutical Sciences. 8(1), 2020, 1-6.

4. Patel J S, Thakkar D, Patel K N, Patel K J. A Review on Bilayer Tablet, Journal of Drug Discovery and Therapeutics, 1(3), 2013, 40-48.

5. Pawar R, Jaimini M, Bhupendra S, Chauhan, Sharma S K. Compression Coated Tables As A Drug Delivery System (Tablet In Tablet): A Review, International Journal of Pharmaceutical Research and Development, 6(1), 2014, 021-033.

6. Mishra A, Bhatt K G, Kothiyal P. Review: Bilayer Tablet and Evaluation, International Journal of Drug and Research and Technology, 3(2), 2013, 21-30.

7. Patel S J, Thakkar D et al. An Overview on Bilayer Tablet, International Journal of Research and Review in Pharmacy and Applied science, 6(1), 2014, 6-15.

8. Kavitha K, Divya A et al. Bilayer Tablet Technology: An Overview, Journal of Applied Pharmaceutical Sciences, 1(8), 2011, 43-47.

9. Musle K, Payghan S A, Disuza J J. Formulation, Evaluation and Development of Bilayer Tablet, International Journal of Pharmaceutical Research and Development (IJPRD), 3(10), 2011, 80-87.

10. Brito Raj S, Sravani G, Bhanupriya N, Veerupakshi M. Design and Evaluation of Sustained Release Bilayer Tablet of Metformin Hydrochloride with Metoprolol Tartrate, International Journal of Novel Trends in Pharmaceutical Sciences, 1(1), 2011, 1-5.

11. Rajendra $\mathrm{N}$ and Natarajan R. Formulation And Evaluation of Sustained Release Bilayer Tablet Of Metformin HCL And Pioglitazone HCL, International Journal of Current Pharmaceutical Research, 3(3), 2011, 118-122.
12. Nagaraju R and Kaza R. Formulation And Evaluation of Bilayer Sustained Release Tablet Of Salbutamol And Theophylline, International journal of pharmaceutical science and nano technology, 2(3), 2009, 638-646.

13. Veerareddy $\mathrm{P}$ and Manthri R. Formulation and Evaluation of Compression Coated Piroxicam Tablets for Colon Specific Drug Delivery, Acta Pharma, 52(3), 2010, 281294.

14. Durga Prasad Pattanayak and Subas C Dinda. Bilayer Tablet Formulation of Metformin Hydrochloride and Glimepiride: A novel approach to improve therapeutic efficacy, International journal of drug discovery and herbal research, 1(1), 2011, 1-4.

15. Cremer K. Pharmaceutical Application of Layer Tablet, AAPS Pharm Sci. Tech, 2001.

16. Ramana G, Sushma M, Arun Y. Formulation and Evaluation of Sustained Release Bilayer Tablet of Ambroxol Hydrochloride, International Journal Innovative Pharmaceutical Research, 1(3), 2010, 61-65.

17. Deore R K, Kunchu K, Theeta G. Preparation And Evaluation of Sustained Release Matrix Tablets of Tramadol Hydrochloride, Tropical Journal of Pharmace Research, 9(3), 2010, 275-281.

18. https://en.wikipedia.org.

19. www.google.com.

Please cite this article in press as: Mahesh Dattatray Dokhe et al. Tablet in tablet: a review, Asian Journal of Research in Chemistry and Pharmaceutical Sciences, 8(1), 2020, 1-6. 